\title{
Fine mapping of a QTL for ear size on porcine chromosome 5 and identification of high mobility group AT-hook 2 (HMGA2) as a positional candidate gene
}

Pinghua $\mathrm{Li}^{1 \dagger}$, Shijun Xiao ${ }^{1 \dagger}$, Na Wei ${ }^{1}$, Zhiyan Zhang ${ }^{1}$, Ruihua Huang ${ }^{2}$, Yueqing $\mathrm{Gu}^{3}$, Yuanmei Guo ${ }^{1}$, Jun Ren ${ }^{1}$, Lusheng Huang ${ }^{1}$ and Congying Chen ${ }^{1 *}$

\begin{abstract}
Background: Ear size and shape are distinct conformation characteristics of pig breeds. Previously, we identified a significant quantitative trait locus (QTL) influencing ear surface on pig chromosome 5 in a White Duroc $\times$ Erhualian $\mathrm{F}_{2}$ resource population. This QTL explained more than $17 \%$ of the phenotypic variance.

Methods: Four new markers on pig chromosome 5 were genotyped across this $F_{2}$ population. RT-PCR was performed to obtain expression profiles of different candidate genes in ear tissue. Standard association test, marker-assisted association test and F-drop test were applied to determine the effects of single nucleotide polymorphisms (SNP) on ear size. Three synthetic commercial lines were also used for the association test.
\end{abstract}

Results: We refined the QTL to an 8.7-cM interval and identified three positional candidate genes i.e. HMGA2, SOX5 and PTHLH that are expressed in ear tissue. Seven SNP within these three candidate genes were selected and genotyped in the $F_{2}$ population. Of the seven SNP, HMGA2 SNP (JF748727: g.2836 A > G) showed the strongest association with ear size in the standard association test and marker-assisted association test. With the F-drop test, F value decreased by more than 97\% only when the genotypes of HMGA2 g.2836 A > G were included as a fixed effect. Furthermore, the significant association between g.2836 A > G and ear size was also demonstrated in the synthetic commercial Sutai pig line. The haplotype-based association test showed that the phenotypic variance explained by HMGA2 was similar to that explained by the QTL and at a much higher level than by SOX5. More interestingly, HMGA2 is also located within the dog orthologous chromosome region, which has been shown to be associated with ear type and size.

Conclusions: HMGA2 was the closest gene with a potential functional effect to the QTL or marker for ear size on chromosome 5. This study will contribute to identify the causative gene and mutation underlying this QTL.

\section{Background}

Ear size and erectness are important conformation characteristics of pig breeds. Moreover, ear defects have been observed in different species [1,2]. For example, in humans, congenital underdevelopment of the external ear or microtia, affects about 1 in 6000 children in

\footnotetext{
* Correspondence: chcy75@hotmail.com

+ Contributed equally

${ }^{1}$ Key Laboratory for Animal Biotechnology of Jiangxi Province and the Ministry of Agriculture of China, Jiangxi Agricultural University, Nanchang 330045, People's Republic of China

Full list of author information is available at the end of the article
}

Europe and the USA, and 1 in 4000 in Japan and China [3]. Pig can be used as an animal model to investigate the underlying mechanisms responsible for the development of the external ear. However, few genetic analyses have been carried out to study ear development in pigs and to our knowledge, only three studies have reported data on the mapping of QTL (Quantitative Trait Loci) for pig ear traits. In 2004, Guo et al. (in Chinese) [4] showed the existence of a QTL for ear erectness on pig chromosome 6 or SSC6 (SSC for Sus scrofa) in a commercial population. In 2007, Wei et al.[5] identified two

\section{Biomed Central}


significant QTL with a major effect on ear size on SSC5 and SSC7 in a Large White $\times$ Meishan $\mathrm{F}_{2}$ resource population. Another study involving a White Duroc $x$ Erhualian $\mathrm{F}_{2}$ intercross also detected two major QTL for ear size on the same chromosomes, which explained more than $45 \%$ and $17 \%$ of the phenotypic variance, respectively [6]. Although the genetic positions of the QTL were different (51 cM vs. $70 \mathrm{cM}$ on SSC5 and 70 cM vs. $58 \mathrm{cM}$ on SSC7), the $95 \%$ confidence interval of both QTL overlapped partly in the two studies. More recently, a missense mutation on PPARD was identified as the causative mutation for the QTL on SSC7, which influences ear size by mediating down-regulation of $\beta$ catenin and its target gene expression [7]. Concerning the QTL on SSC5, the confidence interval was too large $(11 \mathrm{cM})$ for positional cloning analysis and the genetic basis of the QTL remains unexplored.

Thus, the study reported here, aimed at investigating the causative gene for ear size on SSC5 by refining the QTL region using higher-density markers on this chromosome and at analyzing in further detail, the association between the three candidate genes located in the refined region with ear size in the White Duroc $x$ Erhualian $\mathrm{F}_{2}$ resource population and three synthetic commercial lines. The results will contribute to the final characterization of the causative gene or mutation responsible for this QTL.

\section{Methods}

\section{Animals and phenotypic measurements}

The White Duroc $\times$ Erhualian $F_{2}$ resource population and phenotypic measurements have been described in detail in Ma et al.[6]. Briefly, the $\mathrm{F}_{2}$ resource population was obtained by mating two White Duroc sires with small and erect ears to 17 Erhualian dams with large and floppy ears to produce $F_{1}$ animals and then by random crossing between nine F1 boars and 59 F1 sows (avoiding sister-brother mating) to produce a total of $1912 F_{2}$ animals. Among the F2 individuals, 1029 were phenotyped by measuring separately the size of both left and right ears after slaughter at 240 days of age as described in our previous report [6].

Three synthetic commercial lines including Sutai (Duroc $\times$ Erhualian, $n=173$ ), Sujiang (Duroc $\times$ Jiangquhai, $\mathrm{n}=105$ ) and Suzhong (Landrace $\times$ Erhualian, $\mathrm{n}=$ 78) were used in association tests to confirm the association between candidate genes and ear size. Both Erhualian and Jiangquhai indigenous breeds have large ears. Measurement of ear size was done according to the method described by Ren et al.[7], which differs from that of Ma et al.[6]. Briefly, the ears of each animal were fixed and photographed with a ruler placed on the surface of the ear as an internal size reference. The surface of each ear was calculated using the Leica Qwin software. All experimental animal procedures were conducted according to the guidelines for the care and use of experimental animals established by the Ministry of Agriculture of China.

\section{Microsatellite genotyping}

Genomic DNA was extracted from ear and spleen tissues according to the routine phenol/chloroform extraction method and diluted to a final concentration of 20 $\mathrm{ng} / \mu \mathrm{L}$. To increase marker density in the QTL candidate region, microsatellite markers were selected from the SWR453-SW1987 interval on the USDA-MARC SSC5 linkage map [8] or by mining genomic sequence data of the QTL region (Sscrofa 9.2) using the SSRHunter program [9]. Four informative microsatellite markers, namely SSC5P3, SSC5P13, SSC5P16 and SW2003, were genotyped in all $\mathrm{F}_{0}, \mathrm{~F}_{1}$ and $1029 \mathrm{~F}_{2}$ animals as described previously [10]. All the primers were designed using the online software Primer3 [11] and primer sequences, annealing temperatures, amplicon lengths and number of alleles are shown in Additional file 1: Table S1.

\section{Selection of candidate genes and RT-PCR}

Orthologous regions between the human genome and the refined QTL region were detected using the pighuman comparative map in the pig QTL database [12]. Annotated genes present in the orthologous regions were identified with the UCSC genome browser [9] and their functions obtained from the NCBI database [13] or GeneCards [14].

SOX5, HMGA2 and PTHLH were selected from the refined QTL region as genes with related functions. SOX5 encodes a member of the SOX (SRY-related HMG-box) transcription factors family, and a previous study has indicated that it plays a role in chondrogenesis $[15,16]$. Moreover, mutations in the genes encoding homeobox transcription factors are responsible for the defective development of outer ears [17]. HMGA2 encodes a protein that belongs to the non-histone chromosomal high mobility group (HMG) protein family. HMGA2-LPP fusion protein promotes chondrogenesis by up-regulating cartilage-specific collagen gene expression through the $\mathrm{N}$-terminal DNA binding domains [18]. The presence of HMGA proteins increases the proliferative activity of chondrocytes cultured in vitro [19]. More interestingly, HMGA2-deficient mice develop smaller ears [20] and in dogs, HMGA2 may be involved in differences in the size and type of ears [21]. PTHLH is a member of the parathyroid hormone family which regulates endochondral bone development through regulation of chondrocyte proliferation and differentiation during early bone growth [22]. The Wnt/beta-catenin pathway interacts differentially with the parathyroid hormone-related protein (PTHRP), which acts as a control 
of chondrocyte hypertrophy and final maturation [23]. Cartilage is the major component of ear tissue and genes influencing chondrocyte proliferation have been shown to affect ear size [24]. Thus, based on both the physiological roles of SOX5, HMGA2 and PTHLH in chondrogenesis and cartilage formation and their expression in ear tissues, we consider that they are important candidate genes for ear size.

RT-PCR was performed to check expression profiles of HMGA2,SOX5 and PTHLH in ear tissue with the $\beta$ actin gene as an internal control. Ear tissues were sampled from two Erhualian piglets at two days of age, immediately frozen in liquid nitrogen and finally conserved at $-80^{\circ} \mathrm{C}$. Total RNA was extracted with the Nucleospin RNA kit (QIAGEN, Hilden, Germany) following instructions in the manual. The first-strand cDNA was synthesized from $2 \mu \mathrm{g}$ of total RNA according to the protocol of Omniscript reverse transcriptase kit (QIAGEN, Hilden, Germany). Primers were designed to amplify regions across the intron/exon boundaries to avoid genomic DNA contamination. Details on primers are in Additional file 1: Table S2. RT-PCR was performed in a $15 \mu \mathrm{L}$ reaction volume with a touchdown PCR protocol.

\section{SNP identification and genotyping}

Genomic DNA of four randomly selected $F_{1}$ boars was used to identify SNP in the candidate genes. All the exons and part of the introns were amplified and sequenced with the primers listed in Additional file 1: Table S3. PCR amplification was carried out with a PE9700 thermal cycler (Applied Biosystem Inc., Drive Foster City, USA). After purification with the QIAquick Gel Extraction kit (Qiagen, Hilden, Germany), PCR products were bidirectionally sequenced on an ABI 3130 XL Genetic Analyzer (ABI, Foster City, USA). The sequences of each amplicon were analyzed with SeqMan in the DNAStar software package.

Informative SNP (i.e. with an allele frequency $\geq 25 \%$ in four boars) were chosen for further genotyping using the SNaPshot kit (Applied Biosystem Inc., Drive Foster City, USA) according to the manufacturer's protocol. Primers for PCR and SNaPshot extension assays are listed in Additional file 1: Table S4. After purification and denaturation at $95^{\circ} \mathrm{C}$ for $5 \mathrm{~min}$, the SNaPshot reaction mixture was separated on an ABI 3130XL genetic analyzer for data collection. The SNP genotypes were recorded and analyzed using the GeneScan version 4.0.

\section{Statistical analysis}

The linkage map of SSC5 was re-constructed by CRIMAP 2.4 [25]. The physical positions of the seven SNP on SSC5 were determined by the SOAP2 tool based on the reference genome assembly of Sscrofa 10.2 [26]. QTL analyses were carried out with QTL Express [27]. The statistical model was similar to the initial QTL mapping model [6]. Briefly, batch and sex were treated as fixed effects, and carcass weight as a covariate. The 95\% confidence interval (CI) was determined using a bootstrap method through 2000 iterations [28]. A multiple QTL model was used to exclude the effects of previously detected QTL on other chromosomes by treating them as fixed effects. The significance threshold was determined with 1000 permutation tests [29].

Because of the extensive between-breed linkage disequilibrium (LD) in the $\mathrm{F}_{2}$ resource populations, standard association test, marker-assisted association test and Fdrop test were performed to analyze the association between the candidate genes and the ear size in the current $\mathrm{F}_{2}$ resource population as described in Zhao et al. [30]. Briefly, the following models were used:

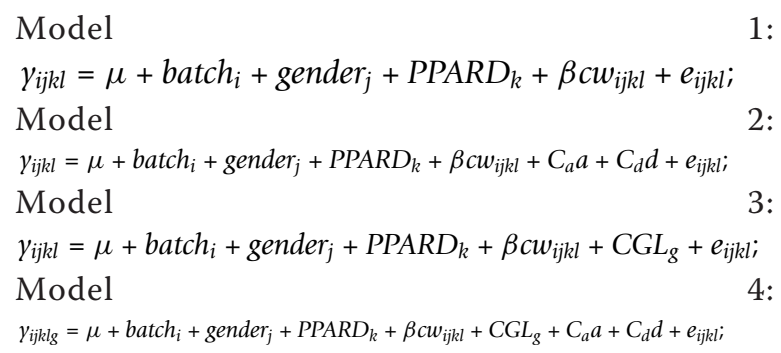

where $y_{i j k l}$ is the observation for ear size; $\mu$ is the overall mean for ear size; batch $_{i}$ is the fixed effect of the $\mathrm{i}^{\text {th }}$ batch $(\mathrm{i}=1,2,3,4,5,6)$; gender $_{j}$ is the fixed effect of the $\mathrm{j}^{\text {th }} \operatorname{sex}(\mathrm{j}=1,2) ; P P A R D_{k}$ is the fixed effect of the $\mathrm{k}^{\mathrm{ith}}$ genotype of PPARD $(\mathrm{k}=1,2,3) ; C G L_{g}$ is the $\mathrm{g}^{\text {th }}$ genotype effect of the candidate gene $(\mathrm{g}=1,2,3) ; \beta$ is the regression coefficient for carcass weight; $c w_{i j k l}$ is the covariate of the carcass weight; a and $d$ are unknown additive and dominance effects of average QTL alleles by breed origin from the two $F_{0}$ breeds, respectively, and $C_{a}$ and $C_{d}$ are the additive and dominance coefficients, as computed from marker information [31]; and $e_{i j k l}$ is the residual. The standard association test was based on an F ratio of residual sums of squares (RSS) for models 1 and 3. An F ratio reaching or exceeding the significant threshold means that the candidate gene is in LD with the QTL or is the QTL. The markerassisted association test for candidate genes was based on an F ratio of RSS for models 4 and 2. In this case, if the $\mathrm{F}$ ratio reaches or exceeds the significant threshold, then the candidate gene locus can account for most of the phenotype variance effect and it is in LD with the quantitative trait nucleotide (QTN) or is the QTN [30]. The marker-assisted association test removed the impact of between-breed LD. The F-drop test was based on 
fitting the models 4 and 3 . If a candidate gene that is in LD with the QTL is included as a fixed effect, it is expected to absorb part of the between-breed QTL effect, and the F-ratio for the QTL is expected to drop. All the tests were carried out with GridQTL version $2.0 .0[32]$.

The haplotype phases were inferred using AlphaPhase [33]. The phenotypes were corrected for sex, batch, carcass weight and additive infinitesimal effects using Qxpak 5 [34]. The residuals were regressed on the number of copies of each haplotype allele separately across all the individuals through the GLM function in R. The $P$ values were corrected for the number of haplotypes (Bonferroni correction). The phenotypic variance explained by all the haplotypes was estimated by the ANOVA function in $\mathrm{R}$. The association of HMGA2 JF748727: g.2836 A > G with ear size in three synthetic commercial lines was evaluated by the GLM procedure of SAS 9.0 (SAS ${ }^{\circledR}$ Institute Inc. Cary, NC). The stratification effect of the population was excluded in the analysis.

\section{Results}

\section{Fine-mapping of the QTL}

To refine the linkage map of the region previously reported on SSC5, four additional microsatellite markers (SSC5P3, SW2003, SSC5P13 and SSC5P16) were identified and genotyped on $F_{0}, F_{1}$ and $1029 F_{2}$ pigs. Thus, the complete linkage map with distances between markers in cM is the following: Acr - 3.0 - SW413 - 40.0 SWR453 - 5.4 - SSC5P3 - 12.3 - SW1904 - 10.3 SW2003 - 2.7 - SSC5P13 - 1.6 - SSC5P16 - 10.9 SW1987 - 21.1 - SW986 - 7.9 - SW995. This new round of QTL mapping reduced the confidence interval of the initial $11 \mathrm{cM}$ QTL region between markers SWR453 and SW1987 to an $8.7 \mathrm{cM}$ interval between markers SSC5P3 and $S W 2003$. One thousand permutation tests were carried out and the bootstrap histograms are shown in Figure 1. The most likely position of the QTL is located in a $8.7 \mathrm{cM}$ region (between positions $55.6 \mathrm{cM}$ and 64.3 $\mathrm{cM})$. Three genes with related functions i.e. $H M G A 2$, SOX5 and PTHLH are located within this refined QTL interval. RT-PCR showed that the three genes are expressed in ear tissue (Additional file 2: Figure S1).

\section{Polymorphisms in candidate genes}

We detected 19 SNP and four indels in the HMGA2 gene (accession number: JF748727, Additional file 1: Table S3). None of these polymorphisms were located in the coding region. We found 28 polymorphisms including 27 SNP and one indel in the SOX5 gene (accession number: JF748729, Additional file 1: Table S3) and among all these polymorphisms, only one synonymous SNP was detected in exon 2. A total of 20

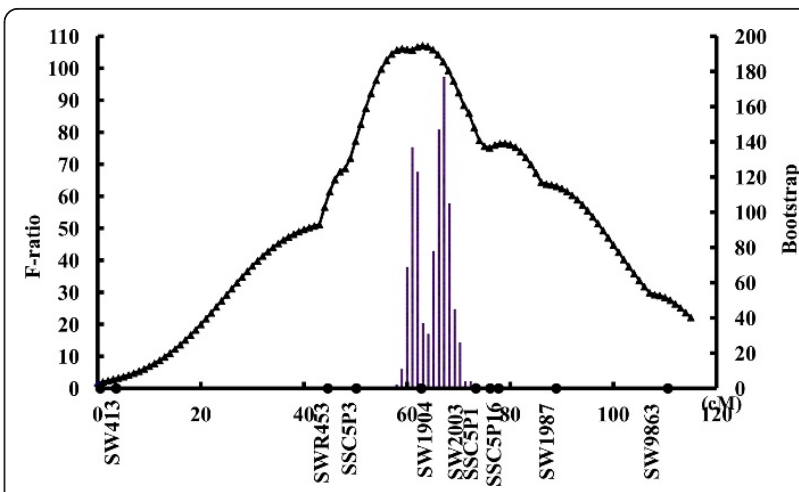

Figure 1 F-value curve and bootstrap histograms of the QTL on SSC5 for ear size. The left Y-axis indicates the F-value in QTL fine-mapping and the right $Y$-axis shows the number of iterations in bootstrapping.

SNP and one indel were identified in the PTHLH gene including one missense mutation in exon 2 (accession number: JF748728, Additional file 1: Table S3). Although a number of mutations were identified in the three candidate genes, most of them were identified in only one animal and thus were not informative and not useful for association tests on the $\mathrm{F}_{2}$ resource population. Finally, only seven SNP for which the minor allele frequency was $>25 \%$ were chosen for further genotyping across the entire White Duroc $\times$ Erhualian intercross.

\section{Association of candidate genes with ear size in the $F_{2}$ resource population}

The physical positions of the seven SNP on the reference genome assembly 10.2 were $32.483 \mathrm{Mb}, 32.486$ $\mathrm{Mb}, 32.497 \mathrm{Mb}, 46.968 \mathrm{Mb}, 51.430 \mathrm{Mb}, 51.431 \mathrm{Mb}$ and $51.480 \mathrm{Mb}$, respectively and were all situated within the interval between SSC5P3 (18.299 Mb) and SW2003 (54.594 Mb). Among the seven SNP, three included within the HMGA2 gene are located near the SW1904 marker $(33.850 \mathrm{Mb})$, which is in the middle of the QTL. Standard association test, marker-assisted association test and F-drop test were carried out to evaluate the association between the seven informative SNP and ear size in the $\mathrm{F}_{2}$ resource population. In the standard association test, all the SNP were in strong association with ear size $\left(P_{-s a}<0.01\right.$, Table 1$)$. HMGA2 g.2836 A > G showed the strongest association with ear surface. Allele [G] present in Erhualian pigs is associated with an increase in ear size, while allele [A] is responsible for a smaller ear surface i.e. $32.84 \pm 5.46 \mathrm{~cm}^{2}$ (Table 1 ).

In the marker-assisted association test, apart from the SNP SOX5 g.12328 T > G that did not achieve a $P<$ 0.05 significance level, the SNP analyzed for the three candidate genes were significantly associated with ear size $\left(P_{-m a}<0.05\right.$, Table 1$)$. Similar to the standard 
Table $1 P$ values obtained in standard association test and marker-assisted association test and additive effects of the seven SNP

\begin{tabular}{|c|c|c|c|c|c|}
\hline Gene & Polymorphism & $P_{-s a}$ & $P_{-m a}$ & Allele & Additive effect \pm s.e. $\left(\mathrm{cm}^{2}\right)$ \\
\hline \multirow[t]{3}{*}{ HMGA2 (JF748727) } & g.2836 A > G & $9.19 \mathrm{E}-46$ & $1.92 \mathrm{E}-05$ & A & $-32.84 \pm 5.46$ \\
\hline & $g .215 C>T$ & $5.04 \mathrm{E}-26$ & $1.23 \mathrm{E}-03$ & $\mathrm{~T}$ & $27.40 \pm 5.37$ \\
\hline & g.1799 G > A & 1.60E-19 & 0.04 & G & $22.54 \pm 4.76$ \\
\hline \multirow[t]{3}{*}{ SOX5 (JF748729) } & g.9084 T > C & $3.51 \mathrm{E}-07$ & 0.03 & C & $32.56 \pm 7.25$ \\
\hline & g.9908 T > C & 4.54E-25 & 0.02 & C & $15.72 \pm 5.00$ \\
\hline & g.12328 T > G & $8.12 \mathrm{E}-07$ & 0.11 & G & $-15.28 \pm 5.02$ \\
\hline PTHLH (JF748728) & g.9440 T > C & 5.12E-07 & 0.03 & C & $11.74 \pm 5.15$ \\
\hline
\end{tabular}

$P_{- \text {-sa }}: P$ values in standard association analysis; $P_{-m a}: P$ values in marker-assisted association analysis; s.e.: standard error; the negative values indicate decrease in ear size

association test, HMGA2 g.2836 A > G was most strongly associated with ear size $\left(P_{-m a}=1.92 \mathrm{E}-05\right)$. The results of the F-drop test are shown in Figure 2. When the genotypes of HMGA2 g.2836 A > G were included as a fixed effect in the QTL analysis, the $\mathrm{F}$ value dropped from 134.14 to 3.19. F values of the other SNP were below $90 \%$.

HMGA2 and SOX5 haplotypes were constructed and their associations with ear size were also analyzed in this study. Overall, the phenotypic variance explained by HMGA2 haplotypes reached $19.47 \%(P=1.31 \mathrm{E}-31$, Table 2). Haplotypes [GCA] and [GTG] were associated with an increase in ear size. Five SOX5 haplotypes showed a significant association with ear size. Animals with haplotype [TCT], [TCG] or [CCT] showed larger phenotypic values than those with haplotype [TTT] or [TTG] (Table 2). However, the phenotypic variance explained by SOX5 haplotypes was lower

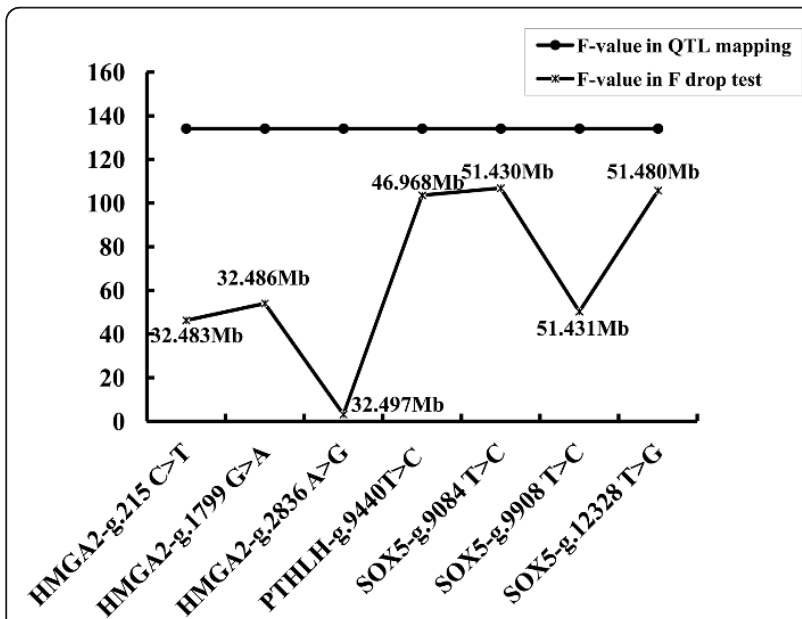

Figure 2 F-value curves of seven SNP in the F-drop test when each SNP was included as a fixed effect. The $Y$-axis shows Fvalues and the $X$-axis indicates the SNP order; $F$ (QTL): $F$ value in QTL mapping (F-values of the model 3), F (drop): F value when the candidate SNP was included as a fixed effect in QTL mapping (values of the F-ratios between models 4 and 3 ) than that of the QTL or HMGA2 haplotypes (12.82\%, $P=2.48 \mathrm{E}-22$ ).

\section{$G$ with ear size in outbred}

populations", 1,0,1,0,0pc,0pc,0pc,0pc>Association of HMGA2 g.2836 A > G with ear size in outbred populations

Given that the strongest association between HMGA2 g.2836 A > G and ear size was identified in the White Duroc $\times$ Erhualian $\mathrm{F}_{2}$ resource population, this SNP was further genotyped in 356 adult pigs from the synthetic commercial lines of Sutai, Sujiang and Suzhong. The results showed that it was significantly associated with ear size in Sutai pigs $(P<0.05)$, but not in Sujiang and Suzhong pigs (Table 3) and allele [G] was responsible for an increase in ear size.

\section{Discussion}

Identification of causative genes underlying QTL in domestic animals is still a major challenge because confidence intervals of QTL are typically too large for positional cloning of the underlying gene [35]. In this study, we refined the interval containing the QTL responsible for ear size on SSC5 from an $11 \mathrm{cM}$ interval to an 8.7 $\mathrm{cM}$ region. However, the confidence interval of the refined QTL is still large. The linkage disequilibriumbased haplotype-sharing approach has been shown to be effective in fine mapping of QTL in domestic animals $[35,36]$. Unfortunately, in our study QTL genotypes could be deduced for only three of the nine $F_{1}$ boars by the marker-assisted segregation analysis (data not shown) and no obvious shared haplotype was observed, which hampered further mapping of the QTL.

In this study, the refined QTL region corresponds to two orthologous regions on human chromosome 12 (65.70-75.07 $\mathrm{Mb}$ and 19.80-33.72 Mb), in which three candidate genes with related functions i.e. SOX5, HMGA2 and PTHLH were identified. The standard association test, marker-assisted association test and Fdrop test were used to test the association between these candidate genes and ear size in the $F_{2}$ resource 
Table 2 Associations of HMGA2 and SOX5 haplotypes with ear size in the $\mathrm{F}_{2}$ resource population

\begin{tabular}{|c|c|c|c|c|}
\hline Gene & Haplotype & Frequency & Effect \pm s.e. ${ }^{*}\left(\mathrm{~cm}^{2}\right)$ & Corrected $P$-value \\
\hline \multirow[t]{8}{*}{ HMGA2 } & $\mathrm{A} / \mathrm{C} / \mathrm{G}$ & 0.002 & $-41.43 \pm 28.64$ & 1 \\
\hline & $\mathrm{A} / \mathrm{C} / \mathrm{A}$ & 0.012 & $-5.09 \pm 9.66$ & 1 \\
\hline & $\mathrm{A} / \mathrm{T} / \mathrm{G}$ & 0.484 & $-20.96 \pm 1.70$ & 4.34E-31 \\
\hline & $\mathrm{A} / \mathrm{T} / \mathrm{A}$ & 0.004 & $-36.21 \pm 15.33$ & 0.15 \\
\hline & $\mathrm{G} / \mathrm{C} / \mathrm{G}$ & 0.001 & $8.73 \pm 28.68$ & 1 \\
\hline & $\mathrm{G} / \mathrm{C} / \mathrm{A}$ & 0.255 & $22.71 \pm 2.22$ & $3.87 \mathrm{E}-22$ \\
\hline & $\mathrm{G} / \mathrm{T} / \mathrm{G}$ & 0.213 & $16.66 \pm 2.71$ & $9.71 \mathrm{E}-09$ \\
\hline & $\mathrm{G} / \mathrm{T} / \mathrm{A}$ & 0.029 & $3.72 \pm 6.66$ & 1 \\
\hline \multicolumn{3}{|c|}{ phenotypic variance explained by all haplotypes } & $19.47 \%$ & $1.31 \mathrm{E}-31$ \\
\hline \multirow[t]{8}{*}{ sox5 } & $\mathrm{T} / \mathrm{T} / \mathrm{T}$ & 0.170 & $-7.99 \pm 2.69$ & 0.03 \\
\hline & $\mathrm{T} / \mathrm{C} / \mathrm{T}$ & 0.234 & $11.09 \pm 2.33$ & $1.83 \mathrm{E}-05$ \\
\hline & $\mathrm{T} / \mathrm{T} / \mathrm{G}$ & 0.359 & $-17.75 \pm 1.96$ & $6.45 \mathrm{E}-18$ \\
\hline & $\mathrm{T} / \mathrm{C} / \mathrm{G}$ & 0.101 & $11.85 \pm 2.97$ & $5.66 \mathrm{E}-04$ \\
\hline & $\mathrm{C} / \mathrm{T} / \mathrm{T}$ & 0.002 & $45.88 \pm 19.90$ & 0.17 \\
\hline & $\mathrm{C} / \mathrm{C} / \mathrm{T}$ & 0.114 & $15.19 \pm 2.96$ & $2.71 \mathrm{E}-06$ \\
\hline & $\mathrm{C} / \mathrm{T} / \mathrm{G}$ & 0.008 & $3.87 \pm 10.37$ & 1 \\
\hline & $\mathrm{C} / \mathrm{C} / \mathrm{G}$ & 0.012 & $17.88 \pm 8.99$ & 0.38 \\
\hline \multicolumn{3}{|c|}{ phenotypic variance explained by all haplotypes } & $12.82 \%$ & $2.48 \mathrm{E}-22$ \\
\hline
\end{tabular}

Effect \pm s.e. represent the effect of haplotypes and its standard error, the negative values indicate decrease in ear size

population in which extensive linkage disequilibrium exists [30]. HMGA2 g.2836 A > G showed the strongest association with ear size both in standard association test and marker-assisted association test. Allele [G] originating from Erhualian pigs was responsible for the increase in ear size, which is consistent with the breed's characteristic large and floppy ears. With the F-drop test, $\mathrm{F}$ value dropped by more than $97 \%$ only if the HMGA2 g.2836 A > G genotype was included as a fixed effect. The phenotypic variance explained by the HMGA2 haplotypes was a little higher than that by the QTL (19.47\% vs.17.14\%). This could be due to the elimination in the association tests of the effect of the causative mutation in PPARD involved in the other major QTL for ear size on SSC7. However, the phenotypic

Table 3 Association of HMGA2 g.2836 A > G with ear size in synthetic commercial lines

\begin{tabular}{llll}
\hline Population & Genotype & $\mathbf{N}$ & Mean \pm s.d.* $\left(\mathbf{c m}^{\mathbf{2}}\right)$ \\
\hline Sutai & {$[\mathrm{AA}]$} & 90 & $258.57 \pm 40.29^{\mathrm{a}}$ \\
& {$[\mathrm{AG}]$} & 76 & $292.60 \pm 46.28^{\mathrm{b}}$ \\
\multirow{3}{*}{ Sujiang } & {$[\mathrm{GG}]$} & 7 & $323.50 \pm 37.96^{\mathrm{c}}$ \\
& {$[\mathrm{AA}]$} & 57 & $252.73 \pm 45.90$ \\
& {$[\mathrm{AG}]$} & 41 & $265.14 \pm 41.23$ \\
Suzhong & {$[\mathrm{GG}]$} & 7 & $280.23 \pm 45.87$ \\
& {$[\mathrm{AA}]$} & 47 & $216.61 \pm 38.34$ \\
& {$[\mathrm{AG}]$} & 28 & $224.55 \pm 59.25$ \\
& {$[\mathrm{GG}]$} & 3 & $226.68 \pm 80.23$ \\
\hline
\end{tabular}

*phenotypes were corrected for sex, batch and farm; the mean values marked with different superscript letter $a, b$ and $c$ indicate significant phenotype differences between each genotype $(P<0.05)$ variance explained by SOX5 haplotypes is much lower than that by the QTL (12.82\% vs. 17.14\%). Furthermore, the statistical models used in this study can be applied to determine which candidate gene is closest to the QTL. If HMGA2 is more strongly associated with the QTL than SOX5 and PTHLH, the residual sum of the squares of HMGA2 (RSS (HMGA2)) in model 3 is expected to be smaller than that of RSS (SOX5) and RSS $(P T H L H)$ [30]. We found that the ratios of RSS (HMGA2)/RSS (SOX5) and RSS (HMGA2)/RSS $(P T H L H)$ were $<1$ ( 0.79 and 0.78 , respectively). These results suggest that $H M G A 2$ is the gene closest to the QTL.

The significant association between HMGA2 g.2836 A $>\mathrm{G}$ and ear size was confirmed in the synthetic commercial line examined in this study. The founder animals of this line and the White Duroc $\times$ Erhualian $F_{2}$ resource population originate from the same pig breeds, i.e. Duroc and Erhualian pigs. However, no significant association was detected in the Sujiang and Suzhong pig populations, which is most likely due to: 1) the different genetic background of the populations; 2) this SNP is only in LD with the causative mutation. Because recombination exists between the SNP and the causative mutation in the Suzhong and Sujiang populations, no significant association can be detected.

More interestingly, an across-breed genome-wide association study (GWAS) in dogs showed that the SNP most strongly associated with dropping ears and ear size is located $98 \mathrm{~kb}$ apart from each other between the $M S R B 3$ and HMGA2 genes [37]. In addition, an 
association between $H M G A 2$ and ear type has been described in dogs [21] and HMGA2-deficient mice are known to have small ears [20]. Combined with the results obtained in this study, we suggest that HMGA2 is the closest gene to the QTL or the marker for ear size on SSC5 in pigs. More refined mapping of the QTL is needed to demonstrate that HMGA2 is responsible for ear size in pig.

\section{Conclusions}

We have fine-mapped a QTL affecting ear size on SSC5 to an 8.7-cM region. Candidate gene analyses suggest that HMGA2 is the closest gene with a potential functional effect to the QTL or marker for ear size on SSC5. These results will contribute to the identification of the causative mutation for this QTL.

\section{Additional material}

Additional_file_ 1: Primers for QTL fine-mapping, gene expression analyses, and SNP identification and genotyping. Table S1 contains primer sequences, annealing temperatures and amplicons of the microsatellite markers, Table $\mathbf{S 2}$ primers for gene expression analyses, Table S3 sequences and assay conditions of the primers for SNP identification and Table S4 sequences and assay conditions of the primers for SNP genotyping.

Additional_file_2: The expression profiles of three candidate genes in ear tissue by RTPCR. Figure S1 shows the expression level of HMGA2, SOX5 and PTHLH in ear tissue obtained by RT-PCR.

\section{Acknowledgements}

This work was supported by the Jiangxi Project for Cultivating Young Scientists, Key project for scientific research from Ministry of Education of China and Natural Science Foundation of Jiangxi Province (2009GQN0032).

\section{Author details \\ ${ }^{1}$ Key Laboratory for Animal Biotechnology of Jiangxi Province and the Ministry of Agriculture of China, Jiangxi Agricultural University, Nanchang 330045, People's Republic of China. ${ }^{2}$ College of Animal Science and Technology, Nanjing Agricultural University, Nanjing 210095, People's Republic of China. ${ }^{3}$ Professional Society for Erhualian Pig Production of Jiaoxi, Changzhou 213116, People's Republic of China.}

\begin{abstract}
Authors' contributions
PHL performed the experiments and wrote the manuscript; SJX collected the samples and performed the experiments; NW performed the experiments; $Z Y Z$ analyzed the data; $\mathrm{RHH}$ and YQG collected the samples; YMG analyzed the data; JR, provided comments and revisions for the manuscript; LSH conceived and designed the experiments and revised the manuscript; CYC performed the experiments, analyzed the data, wrote and revised the manuscript. All authors read and approved the final manuscript.
\end{abstract}

\section{Competing interests}

The authors declare that they have no competing interests.

Received: 10 September 2011 Accepted: 15 March 2012 Published: 15 March 2012

\section{References}

1. Djorbineva MK, Aleksieva SA, Lauvergne JJ: Hereditary shortening of the external ear in Karakachan sheep of Bulgaria. Brief report (1). Rec Med Vet 1985, 161:57-58.
2. Bongers EM, Opitz JM, Fryer A, Sarda P, Hennekam RC, Hall BD, Superneau DW, Harbison M, Poss A, van Bokhoven H, Hamel BC, Knoers NV: Meier-Gorlin syndrome: report of eight additional cases and review. Am J Med Genet 2001, 102:115-124.

3. Aguilar EF: Auricular reconstruction in congenital anomalies of the ear. Facial Plast Surg Clin North Am 2001, 9:159-169.

4. Guo XL, Looft C, Reinsch N, Ernst K: QTL mapping for ear shape based on a commercial pig population. Yi Chuan Xue Bao 2004, 31:819-821.

5. Wei WH, de Koning DJ, Penman JC, Finlayson HA, Archibald AL, Haley CS: QTL modulating ear size and erectness in pigs. Anim Genet 2007, 38:222-226.

6. Ma J, Qi W, Ren D, Duan Y, Qiao R, Guo Y, Yang Z, Li L, Milan D, Ren J, Huang $L$ : A genome scan for quantitative trait loci affecting three ear traits in a White Duroc $\times$ Chinese Erhualian resource population. Anim Genet 2009, 40:463-467.

7. Ren J, Duan YY, Qiao RM, Yao F, Zhang ZY, Yang B, Guo YM, Xiao SJ, Wei RX, Ouyang ZX, Ding NS, Ai HS, Huang LS: A missense mutation in PPARD causes a major QTL effect on ear size in pigs. PLoS Genet 2011, 7: e1002043.

8. Rohrer GA, Alexander LJ, Hu Z, Smith TP, Keele JW, Beattie CW: A comprehensive map of the porcine genome. Genome Res 1996, 6:371-391.

9. UCSC Pig Genome database. [http://genome.ucsc.edu/cgi-bin/hgGateway].

10. Guo Y, Mao H, Ren J, Yan X, Duan Y, Yang G, Ren D, Zhang Z, Yang B, Ouyang J, Brenig B, Haley C, Huang L: A linkage map of the porcine genome from a large-scale White Duroc $\times$ Erhualian resource population and evaluation of factors affecting recombination rates. Anim Genet 2009, 40:47-52.

11. Primer 3. [http://frodo.wi.mit.edu/primer3/].

12. Pig QTL database. [http://www.animalgenome.org/cgi-bin/QTLdb/SS/ index].

13. NCBI. [http://www.ncbi.nlm.nih.gov/].

14. GeneCards. [http://www.genecards.org/].

15. Lefebvre V, Li P, de Crombrugghe B: A new long form of Sox5 (L-Sox5), Sox6 and Sox9 are coexpressed in chondrogenesis and cooperatively activate the type II collagen gene. EMBO J 1998, 17:5718-5733.

16. Smits P, Li P, Mandel J, Zhang Z, Deng JM, Behringer RR, de Crombrugghe B, Lefebvre V: The transcription factors L-Sox5 and Sox6 are essential for cartilage formation. Dev Cell 2001, 1:277-290.

17. Fekete DM: Development of the vertebrate ear: insights from knockouts and mutants. Trends Neurosci 1999, 22:263-269.

18. Kubo T, Matsui Y, Goto T, Yukata K, Yasui N: Overexpression of HMGA2LPP fusion transcripts promotes expression of the alpha 2 type $\mathrm{XI}$ collagen gene. Biochem Biophys Res Commun 2006, 340:476-481.

19. Richter A, Hauschild G, Murua Escobar H, Nolte I, Bullerdiek J: Application of highmobility-group-A proteins increases the proliferative activity of chondrocytes in vitro. Tissue Eng Part A 2009, 15:473-477.

20. Xiang X, Benson KF, Chada K: Mini-mouse: disruption of the pygmy locus in a transgenic insertional mutant. Science 1990, 247:967-969.

21. Boyko AR, Quignon P, Li L, Schoenebeck JJ, Degenhardt JD, Lohmueller KE, Zhao K, Brisbin A, Parker HG, vonHoldt BM, Cargill M, Auton A, Reynolds A, Elkahloun AG, Castelhano M, Mosher DS, Sutter NB, Johnson GS, Novembre J, Hubisz MJ, Siepel A, Wayne RK, Bustamante CD, Ostrander EA: A simple genetic architecture underlies morphological variation in dogs. PLOS Biol 2010, 8:e1000451.

22. Tenne M, McGuigan F, Jansson L, Gerdhem P, Obrant KJ, Luthman H, Akesson K: Genetic variation in the PTH pathway and bone phenotypes in elderly women: evaluation of PTH, PTHLH, PTHR1 and PTHR2 genes. Bone 2008, 42:719-727.

23. Guo X, Mak KK, Taketo MM, Yang Y: The Wnt/beta-catenin pathway interacts differentially with PTHrP signaling to control chondrocyte hypertrophy and final maturation. PLoS One 2009, 4:e6067.

24. Kusuhara $H$, Isogai $N$, Enjo M, Otani $H$, Ikada $Y$, Jacquet $R$, Lowder $E$, Landis WJ: Tissue engineering a model for the human ear: assessment of size, shape, morphology, and gene expression following seeding of different chondrocytes. Wound Repair Regen 2009, 17:136-146.

25. Green P, Falls K, Crooks S: Documentation for CRIMAP, Version 2.4. Washington University School of Medicine, St Louis, MO; 1990.

26. Li R, Yu C, Li Y, Lam TW, Yiu SM, Kristiansen K, Wang J: SOAP2: an improved ultrafast tool for short read alignment. Bioinformatics 2009, 25:1966-1967. 
27. Seaton G, Haley CS, Knott SA, Kearsey M, Visscher PM: QTL Express: mapping quantitative trait loci in simple and complex pedigrees. Bioinformatics 2002, 18:339-340.

28. Visscher PM, Thompson R, Haley CS: Confidence intervals for QTL locations using bootstrapping. Genetics 1996, 143:1013-1020

29. Churchill GA, Doerge RW: Empirical threshold values for quantitative trait mapping. Genetics 1994, 138:963-971.

30. Zhao H, Rothschild MF, Fernando RL, Dekkers JC: Tests of candidate genes in breed cross populations for QTL mapping in livestock. Mamm Genome 2003, 14:472-482.

31. Haley CS, Knott SA, Elsen JM: Mapping quantitative trait loci in crosses between outbred lines using least squares. Genetics 1994, 136:1195-1207.

32. Seaton G, Hernandez J, Grunchec JA, White I, Allen J, De Koning DJ, Wei W, Berry D, Haley C, Knott S: GridQTL: a grid portal for QTL mapping of compute intensive datasets. Proceedings of the 8th World Congress on Genetics Applied to Livestock Production Belo Horizonte 2006.

33. AlphaPhase. [http://sites.google.com/site/hickeyjohn/alphaphase].

34. Pérez-Enciso M, Misztal I: Qxpak.5: old mixed model solutions for new genomics problems. BMC Bioinformatics 2011, 12:202.

35. Georges M: Mapping, fine mapping, and molecular dissection of quantitative trait loci in domestic animals. Annu Rev Genomics Hum Genet 2007, 8:131-162.

36. Nezer C, Collette C, Moreau L, Brouwers B, Kim JJ, Giuffra E, Buys N, Andersson L, Georges M: Haplotype sharing refines the location of an imprinted quantitative trait locus with major effect on muscle mass to a 250-kb chromosome segment containing the porcine IGF2 gene. Genetics 2003, 165:277-285.

37. Vaysse A, Ratnakumar A, Derrien T, Axelsson E, Rosengren Pielberg G, Sigurdsson S, Fall T, Seppälä EH, Hansen MS, Lawley CT, Karlsson EK, LUPA Consortium, Bannasch D, Vilà C, Lohi H, Galibert F, Fredholm M, Häggström J, Hedhammar A, André C, Lindblad-Toh K, Hitte C, Webster MT: Identification of genomic regions associated with phenotypic variation between dog breeds using selection mapping. PLoS Genet 2011, 7: e1002316.

doi:10.1186/1297-9686-44-6

Cite this article as: Li et al:: Fine mapping of a QTL for ear size on porcine chromosome 5 and identification of high mobility group AThook $2(H M G A 2)$ as a positional candidate gene. Genetics Selection Evolution 2012 44:6.

\section{Submit your next manuscript to BioMed Central and take full advantage of:}

- Convenient online submission

- Thorough peer review

- No space constraints or color figure charges

- Immediate publication on acceptance

- Inclusion in PubMed, CAS, Scopus and Google Scholar

- Research which is freely available for redistribution

Submit your manuscript at www.biomedcentral.com/submit
Biomed Central 